\title{
Inferring phylogeny among cryptic lineages of Eisenia nordenskioldi nordenskioldi (Lumbricidae) based on transcriptomic data
}

\author{
S.V. Shekhovtsov ${ }^{1,2 *}$, N.I. Ershov ${ }^{1}$, G.V. Vasiliev ${ }^{1}$, S.E. Peltek ${ }^{1}$ \\ ${ }^{1}$ Institute of Cytology and Genetics SB RAS, Novosibirsk, Russia \\ ${ }^{2}$ Institute of Biological Problems of the North FEB RAS, Magadan, Russia \\ *e-mail: shekhovtsov@bionet.nsc.ru
}

Key words: earthworms, transcriptomics, phylogeny, cryptic lineages

Motivation and Aim: Cryptic diversity is widespread among little-studied taxa with poor morphologic diversity. In earthworms it is manifested by the presence of strongly diverged (10-20\% of sequence divergence for the COI gene) mitochondrial haplotypes within otherwise morphologically solid species, and even within single populations. Such situation was found in Eisenia nordenskioldi (Eisen, 1879) a widespread Siberian species, in which at least 14 cryptic mitochondrial lineages were found [1].

It proved hard to find out if nuclear genomes of these cryptic lineages are have a similar level of sequence divergence, because many earthworms are polyploids, and thus construction of universal primers, DNA amplification and sequencing often fail. In this study we obtained transcriptomic data for five genetic lineages of E. n. nordenskioldi, the pigmented subspecies of E. nordenskioldi, as well as from the congeneric E. andrei, and attempted to reveal phylogenetic relationships among them.

Methods and Algorithms: Total RNA was extracted from living specimens, poly-A fraction was isolated, reverse transcribed and sequenced using the IonTorrent platform. Reads were assembled using the Trinity software. ORFs and corresponding proteins were predicted using TransDecoder, and CD-HIT was utilized to remove duplicated sequences (95\% similarity threshold). Orthogroups of proteins were defined using ProteinOrtho and aligned using Clustal-Omega aligner. Alignments were strictly trimmed with TrimAl, concatenated and converted to NEXUS format. MrBayes software was used to select an appropriate model (Jones) and infer phylogeny.

Results: We constructed a Bayesian phylogenetic tree for protein sequences of 1185 orthologous sequences with the total alignment length of 190081 a. a. E. n. nordenskioldi proved to be monophyletic. Cryptic lineages of E. n. nordenskioldi had 7.4-7.7\% sequence divergence from the outgroup (p-distance), while intraspecific divergence was as low as $2.2 \%$ between lineages 9 and 7 (from Magadan and Bashkiria, respectively), up to $3.9-5.3 \%$ among the rest of the lineages. Intraspecific divergence within $E$. $n$. nordenskioldi is thus sufficiently high, comparable to that between $E$. n. nordenskioldi and strongly differing congeneric E. andrei.

Conclusion: The obtained results suggest that cryptic lineages of E. nordenskioldi can indeed be considered as separate species.

Acknowledgements: Supported by the RFBR (18-04-00507_a) and by the State Assignment No. 0324-2018-0017.

\section{References}

1. Shekhovtsov S.V., Berman D.I., Bazarova N.E., Bulakhova N.A., Porco D., Peltek S.E. (2016) Cryptic genetic lineages in Eisenia nordenskioldi pallida (Oligochaeta, Lumbricidae). European Journal Soil Biology. 75:151-156. 